\title{
Effects of PPAR- $\alpha$ agonist and IGF-1 on estrogen sulfotransferase in human vascular endothelial and smooth muscle cells
}

\author{
YINTAO $\mathrm{LI}^{1 *}$, YALI XU ${ }^{2,3^{*}}$, XIAOBO $\mathrm{LI}^{3}$, YEJUN QIN ${ }^{2}$ and RENMING HU ${ }^{1}$ \\ ${ }^{1}$ Department of Endocrinology and Metabolism, Institute of Endocrinology and Diabetology, \\ Huashan Hospital, Shanghai Medical College, Fudan University, Shanghai 200040; \\ ${ }^{2}$ Department of Pathology, Provincial Hospital Affiliated to Shandong University, Jinan, Shandong 250021; \\ ${ }^{3}$ Department of Physiology and Pathophysiology, Shanghai Medical College, Fudan University, Shanghai 200032, P.R. China
}

Received January 18, 2013; Accepted April 25, 2013

DOI: $10.3892 / \mathrm{mmr} .2013 .1483$

\begin{abstract}
Estrogen has a protective role in vascular functions and estrogen levels are modulated by estrogen sulfotransferase (SULT1E1). In this study, we investigated the effects of the peroxisome proliferator-activated receptor- $\alpha$ (PPAR- $\alpha$ ) agonist WY14643 and insulin-like growth factor-1 (IGF-1) on the expression and activity of SULT1E1 in vascular cells. Human umbilical vein endothelial cells (HUVECs) and human umbilical artery smooth muscle cells (HUASMCs) were primarily cultured from fresh umbilical cord. SULT1E1 was highly expressed in HUVECs and HUASMCs according to immunofluorescence microscopy detection. WY14643 decreased, while IGF-1 increased, SULT1E1 mRNA and SULT1E1 protein levels, as demonstrated by RT-qPCR and western blot analysis, respectively, in the HUVECs and HUASMCs. SULT1E1 activity was indicated by counting the transformed ${ }^{3} \mathrm{H}$-estradiol sulfate from ${ }^{3} \mathrm{H}$-labeled $17 \beta$-estradiol added into the cell culture medium. The activity of SULT1E1 reduced following treatment with WY14643, whereas SULT1E1 activity was enhanced in the presence of IGF-1. The human SULT1E1 promoter-reporter plasmid was constructed. The activity of the SULT1E1 promoter increased 30 -fold compared with the pGL3-basic vector. The PPAR- $\alpha$ agonist WY14643 downregulated, while IGF-1 upregulated, the luciferase activity of the SULT1E1 promoter. In conclusion, the PPAR- $\alpha$ agonist WY14643 and IGF-1 may regulate SULT1E1 expression at the transcriptional level and modulate the levels of active estrogens in endothelial cells and smooth
\end{abstract}

Correspondence to: Professor Renming $\mathrm{Hu}$, Department of Endocrinology and Metabolism, Institute of Endocrinology and Diabetology, Huashan Hospital, Shanghai Medical College, Fudan University, 12 Middle Wulumuqi Road, Shanghai 200032, P.R. China

E-mail: renminghufudan@gmail.com

*Contributed equally

Key words: estrogen sulfotransferase, peroxisome proliferator-activated receptor- $\alpha$, insulin-like growth factor-1 muscle cells, thereby affecting the physiology and pathophysiology of vascular walls.

\section{Introduction}

There is increasing evidence that supports the theory of an inverse association between plasma estrogen levels and the incidence of cardiovascular disease. The consensus is that estrogen attenuates during the early and advanced stages of atherosclerotic lesion development (1).

Vascular endothelial and smooth muscle cells are important cell types implicated in vascular dysfunction and atherosclerotic plaque development. Estrogens promote endothelial integrity by stimulating the production of nitric oxide and prostacyclin, promoting the proliferation, senescence and migration of endothelial cells, reducing the adhesion of monocytes and neutrophils to the endothelial monolayer and repressing the production of reactive oxygen species. Estrogens have also been shown to inhibit the proliferation and migration of vascular smooth muscle cells, and reduce their inflammatory activation and oxidative stress by increasing the expression of antioxidative enzymes and reducing the expression and activity of NADPH oxidase (1).

Estrogen levels are dependent on the activities of aromatase, $17 \beta$-hydroxysteroid dehydrogenase (17 $\beta$-HSD) type 1 , steroid sulfatase and estrogen sulfotransferase (SULT1E1) (2). Human SULT1E1 is highly efficient at catalyzing the sulfoconjugation of estrone and estradiol at the 3-hydroxyl terminal. As a sulfotransferase (a group of phase II drug-metabolizing enzymes), SULT1E1 was observed to affect tumorigenesis in estrogen-dependent cancers (3), fetal development (4) and adipocyte differentiation (5). Our previous work has demonstrated that SULT1E1 is highly expressed in human umbilical vein endothelial cells (HUVECs) and is important in regulating the inflammatory response and lipid metabolism in endothelial cells (unpublished data).

Several studies have reported aspects of the regulation of SULT1E1. Gong et al (6) reported that activation of nuclear receptor subfamily 3 , group C, member 1 (NR3C1; formerly known as glucocorticoid receptor or GR) by dexamethasone induced the expression and activity of SULT1E1, and the pregnane $X$ receptor (PXR) was reported 
Table I. Primer pairs used to amplify PCR products.

\begin{tabular}{llll}
\hline NCBI gene ID & Gene name & Forward primer (5'-3') & Reverse primer (5'-3') \\
\hline NM_002046.3 & GAPDH & TGTTGCCATCAATGACCCCTT & CTCCACGACGTACTCAGCG \\
NM_005420.2 & SULT1E1 & GGAAGCCATCAGAGGAGC & AAAGTGATTTTTCCAGTCTCC \\
\hline
\end{tabular}

GAPDH, glyceraldehyde 3-phosphate dehydrogenase; SULT1E1, estrogen sulfotransferase.

to repress the expression of the SULT1E1 gene via interactions with hepatocyte nuclear factor $4 \alpha$ (HNF4A) in human primary hepatocytes and hepatocellular carcinoma cells (7).

Peroxisome proliferator-activated receptor- $\alpha$ (PPAR- $\alpha)$, a nuclear receptor that regulates lipid and glucose metabolism, may affect the development and progression of vascular lesions (8). A PPAR- $\alpha$ activator has been observed to reduce the formation of atherosclerotic lesions in humans and in animal models (9-11). PPAR- $\alpha$ is expressed in endothelial and vascular smooth muscle cells, where its effects are anti-inflammatory and anti-atherogenic, and it also has an inhibitory effect on the proliferation and migration of vascular cells (12). Fang et al (13) reported that ciprofibrate (a PPAR- $\alpha$ agonist) increased human SULT2Al (sulfotransferase family, cytosolic, 2A, dehydroepiandrosterone-preferring, member 1) mRNA, immunoreactive protein and enzymatic activity levels by $\sim 2$-fold in primary human hepatocytes. The effect of the PPAR- $\alpha$ agonist on the expression and activity of SULT1E1 remains unknown.

Insulin-like growth factor-1 (IGF-1) is an endocrine and autocrine/paracrine growth factor that exists at high levels in the circulatory system. IGF-1 reduces the atherosclerotic burden and improves features of atherosclerotic plaque stability in animal models via multiple potential mechanisms, including by increasing nitric oxide levels, suppressing plaque cell apoptosis and downregulating tumor necrosis factor (TNF) $(14,15)$. The effect of IGF-1 on the expression and activity of SULT1E1 remains unknown.

As estrogen has a protective role in cardiovascular disease, and the concentration of estrogen is modulated by SULT1E1, understanding the factors that regulate the expression and activity of SULT1E1 is crucial for investigating these diseases. In this study, we investigated the effects of the PPAR- $\alpha$ agonist WY14643 and IGF-1 on the expression and activity of SULT1E1 in HUVECs and human umbilical artery smooth muscle cells (HUASMCs).

\section{Materials and methods}

Culture of HUVECs and HUASMCs. Written informed consent was obtained from patients prior to obtaining HUVECs from fresh umbilical cord veins from normal pregnancies. The study was approved by the ethics committee of Fudan University in China. HUVECs were isolated by collagenase digestion, as previously described (16). HUVECs were cultured in an endothelial cell medium supplemented with $10 \%$ fetal bovine serum, endothelial cell growth supplement and antibiotics. Cells at passage 1-5 were used in the experiments. The HUVECs were identified by their typical 'cobblestone-like' morphology, positive immunofluorescence staining for von Willebrand factor and the uptake of acetylated low-density lipoprotein.

HUASMCs from explants of the human umbilical artery were cultured in M199 medium supplemented with $10 \%$ fetal bovine serum and antibiotics (17). Cells were confirmed as smooth muscle cells by their typical 'hill-and-valley' morphology and positive immunofluorescent staining for $\alpha$-smooth muscle actin ( $\alpha$-SMA). HUASMCs between passages 3 and 7 were used in all experiments. The cells were treated with varying doses of the PPAR- $\alpha$ agonist WY14643 (Sigma-Aldrich, St. Louis, MO, USA) dissolved in DMSO, and IGF-1 (PeproTech, London, UK) dissolved in $\mathrm{ddH}_{2} \mathrm{O}$.

Immunofluorescence detection of SULT1E1 in HUASMCs. HUASMCs were cultured in collagen-coated glass bottom dishes. Cells were washed with PBS, fixed with $3.7 \%$ formaldehyde in PBS for $30 \mathrm{~min}$ at $4^{\circ} \mathrm{C}$ and rinsed three times with PBS at room temperature. Cells were permeabilized in PBS containing $0.2 \%$ Triton X-100 and washed with PBS prior to blocking via incubation with $5 \%$ normal goat serum in PBS for $60 \mathrm{~min}$ at room temperature. Cells were subsequently incubated with $2.5 \%$ normal donkey serum in PBS containing SULT1E1 antibody (1:100 dilution; Proteintech Group, Chicago, IL, USA) or $\alpha$-SMA antibody (1:100 dilution; Boster Biological Technology., Ltd., Fremont, CA, USA) overnight at $4^{\circ} \mathrm{C}$ in an incubator. Cells were washed with PBS containing $0.05 \%$ Tween-20 (3x10 min) and the bound primary antibodies were visualized with fluorescein isothiocyanate (FITC) donkey anti-rabbit IgG or Rhodamine TRITC donkey anti-mouse IgG (Jackson ImmunoResearch Laboratories, Inc., West Grove, PA, USA). After washing, the glass bottom dishes were viewed under a fluorescence microscope. Normal rabbit or mouse IgG served as negative controls.

Determination of SULT1E1 mRNA levels by RT-qPCR assay. Total RNA was extracted using an SV Total RNA Isolation kit (Promega, Madison, WI, USA) according to the manufacturer's instructions. Specific mRNA levels were determined by RT-qPCR assay, as previously described (18). Glyceraldehyde3-phosphate dehydrogenase $(G A P D H)$ was used as an internal standard. Specific primer pairs in the experiment are listed in Table I and referenced in PrimerBank (19).

Determination of SULT1E1 protein levels by western blot analysis. Following treatment, cells were harvested in radioimmunoprecipitation (RIPA) lysis buffer. Proteins were separated by $10 \%$ SDS-PAGE in order to detect the SULT1E1 protein 
level. $\beta$-actin was used as the control. The band densities were quantified by densitometry using the GIS software (Bio-Tanon, Shanghai, China).

Determination of SULT1E1 activity by estrogen sulfation assay. The assay was performed as described by Kushida et al (20). HUASMCs were cultured in 24-well plates with $500 \mu \mathrm{l}$ culture medium, and treated with PPAR- $\alpha$ agonist WY14643 or IGF-1 for $24 \mathrm{~h}$. Subsequently, the cells were incubated with $10,20,30,40,50,60,70,80,90$ or $100 \mathrm{nM}$ cold estrogen combined with $10 \mathrm{nM}{ }^{3} \mathrm{H}$-labeled estradiol $\left(\mathrm{E}_{2} ; 40 \mathrm{Ci} / \mathrm{mmol}\right.$; PerkinElmer, Waltham, MA, USA) for the sulfation assay. Aliquots $(50 \mu \mathrm{l})$ of the medium were sampled at 6 or $24 \mathrm{~h}$, and $50 \mu \mathrm{l}$ of $0.25 \mathrm{M}$ Tris- $\mathrm{HCl}(\mathrm{pH} 8.7)$ and $1 \mathrm{ml}$ of water-saturated chloroform were added to the samples. Following mixing and centrifugation, aliquots $(50 \mu \mathrm{l})$ of the aqueous phase $\left(E_{2} S\right)$ were collected for liquid scintillation counting. SULT1E1 activity was represented by the counts of the aqueous phase.

Construction of human SULT1E1 promoter luciferase reporter vector. To construct the human SULT1E1 promoter-reporter plasmid, genomic DNA was extracted from the HUVECs and amplified by PCR to generate a $1246 \mathrm{bp}$ fragment of the human SULT1E1 5'-flanking sequence. This region spans from positions -1183 to +62 , relative to the upstream region of the transcription start site. The $5^{\prime}$ promoter region of the SULT1E1 gene was replicated with the following primers and subcloned into a luciferase expression vector (pGL3 - basic vector, Promega) between the $K p n I$ and $X h o I$ restriction sites: forward, 5'-GGGGTACCCCTTTTGCATAAGGCTAGATA TTG-3'; and reverse, 5'-CCCTCGAGGGTCTCTTCAAATAC CAAGGCAG-3'. The sequence authenticity of the promoter-reporter plasmid was confirmed by DNA sequencing.

Transient transfection. Promoter-reporter plasmids for transient transfection studies were isolated using a Qiagen plasmid purification kit (Qiagen, Minneapolis, MN, USA). human embryonic kidney 293 (HEK-293) cells were cultured in 6-well plates. Cells were transiently transfected with promoter-reporter plasmids by Lipofectamine ${ }^{\mathrm{TM}} 2000$ (Invitrogen, Carlsbad, CA, USA), according to the manufacturer's instructions. A pRL-CMV plasmid was co-transfected as a transfection efficiency control. The cells were treated, after 24 h, by WY14643 or IGF-1 for a further 24 h. Firefly luciferase and Renilla luciferase activities were quantified using a Dual-Luciferase ${ }^{\circledR}$ reporter assay system (Promega). The relative activity fold-changes were calculated from the results of three independent experiments.

Statistical analysis. Data from three or more separate experiments are presented as the mean \pm SEM. ANOVA and the independent-samples t-test were performed by SPSS 11.0 software (SPSS Inc., Chicago, IL, USA). $\mathrm{P}<0.05$ was considered to indicate a statistically significant difference.

\section{Results}

SULT1E1 washighly expressedinHUASMCs.SULT1E1 expression in HUASMCs was visualized via immunofluorescence.
A

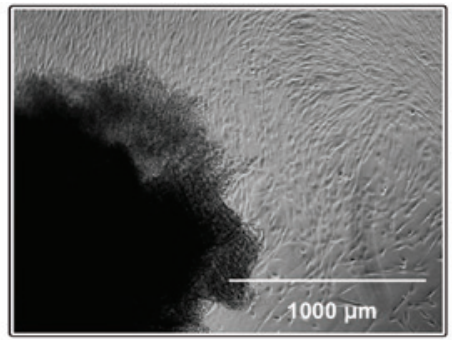

B

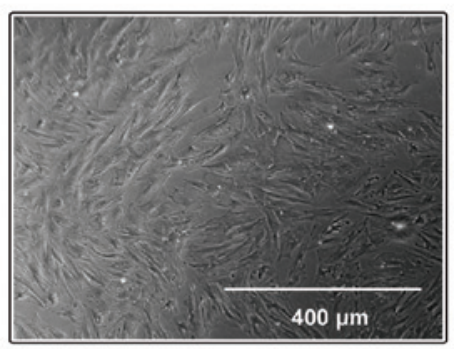

C

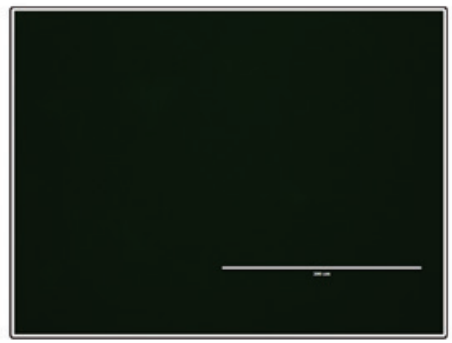

D

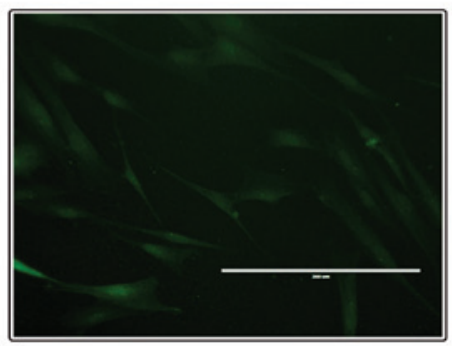

$\mathbf{E}$

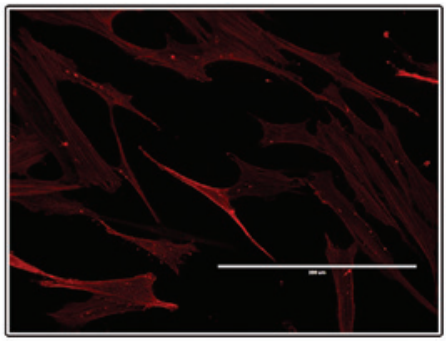

$\mathbf{F}$

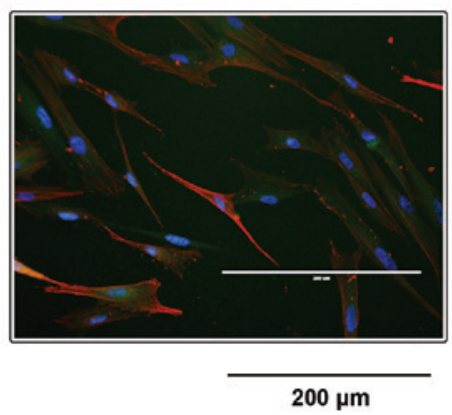

Figure 1. SULT1E1 was highly expressed in HUASMCs. (A) HUASMCs migrated out of the explant from the human umbilical artery in 10 days; (B) HUASMC morphology following passage; (C) negative control; (D) visualization of SULT1E1 in HUASMCs; (E) identification of $\alpha$-SMA in HUASMCs; (F) merged image of SULT1E1 and $\alpha$-SMA in HUASMCs. HUASMCs, human umbilical artery smooth muscle cells; SULTIE1, estrogen sulfotransferase; $\alpha$-SMA, $\alpha$-smooth muscle actin. 
A

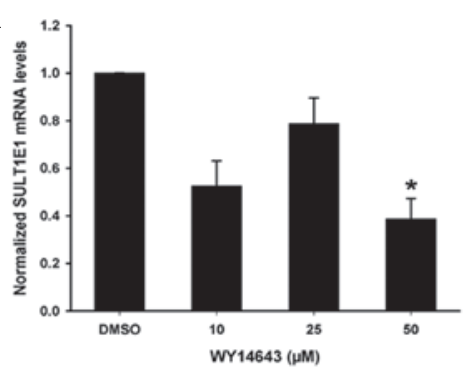

B

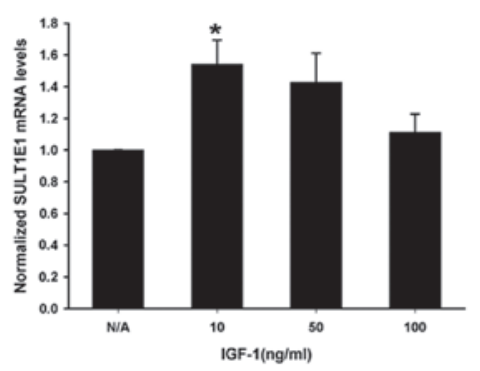

C

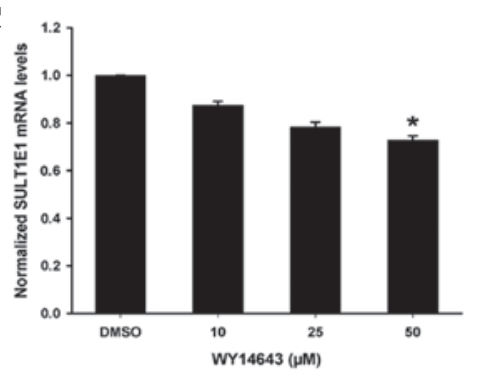

D

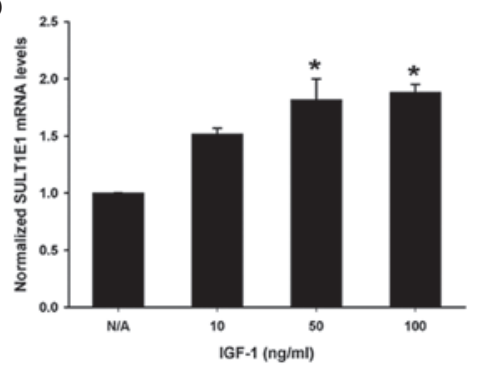

Figure 2. Effects of PPAR- $\alpha$ agonist WY14643 and IGF-1 on SULTIE1 mRNA levels in HUVECs and HUASMCs. (A and B) HUVECs and (C and D) HUASMCs were treated with different doses of (A and C) PPAR- $\alpha$ agonist WY14643 or (B and D) IGF-1 for $24 \mathrm{~h}$. The mRNA levels of SULT1E1 were quantified by RT-qPCR analysis. " $\mathrm{P}<0.05$ compared with the DMSO or N/A group. PPAR- $\alpha$, peroxisome proliferator-activated receptor- $\alpha$; IGF-1, insulin-like growth factor-1; SULTIE1, estrogen sulfotransferase; HUVECs, human umbilical vein endothelial cells; HUASMCs, human umbilical artery smooth muscle cells.

All HUASMCs demonstrated a typical spindle morphology with a 'hill-and-valley' growth pattern (Fig. 1A and B) and had positive reactions to the antibody against $\alpha$-SMA antibody (Fig. 1E). The negative control with normal rabbit IgG exhibited no staining (Fig. 1C). SULT1E1 was highly expressed in HUASMCs (Fig. 1D).

Effects of PPAR- $\alpha$ agonist WY14643 and IGF-1 on SULTIEI mRNA levels in HUVECs and HUASMCs. Different concentrations of WY14643 and IGF-1 were added to the HUVECs (Fig. 2A and B) or HUASMCs (Fig. 2C and D) for 24 h. SULTIE1 mRNA levels were measured by RT-qPCR analysis. WY14643 decreased the SULTIE1 mRNA levels
A

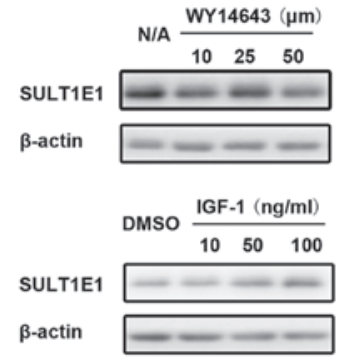

C

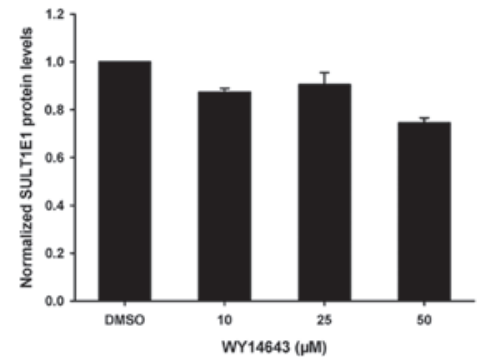

D

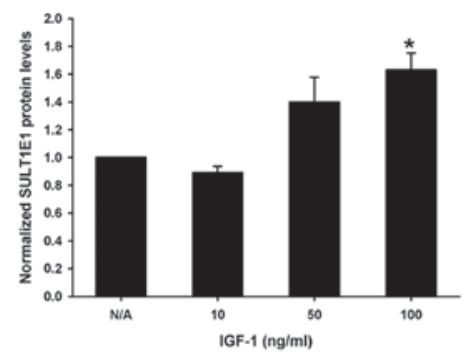

$\mathbf{E}$

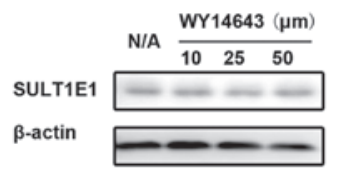

$\mathbf{F}$

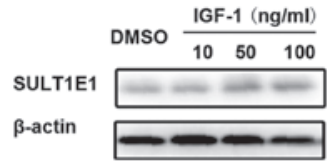

G

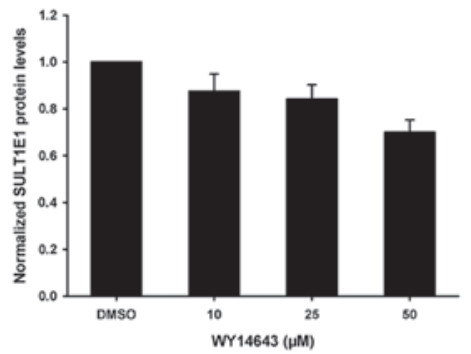

H

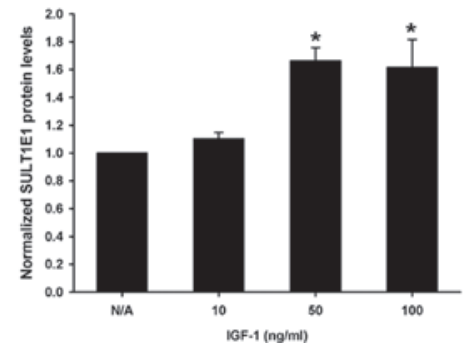

Figure 3. Effects of PPAR- $\alpha$ agonist WY14643 and IGF-1 on the SULT1E1 protein levels in HUVECs and HUASMCs. (A-D) HUVECs and (E-H) HUASMCs were treated with different doses of (A, C, E and G) PPAR- $\alpha$ agonist WY14643 or (B, D, F and H) IGF-1 for $24 \mathrm{~h}$, respectively. The protein levels of SULT1E1 were quantified using western blot analysis. "P<0.05 compared with the DMSO or N/A group. PPAR- $\alpha$, peroxisome proliferator-activated receptor- $\alpha$; IGF-1, insulin-like growth factor-1; SULTIE1, estrogen sulfotransferase; HUVECs, human umbilical vein endothelial cells; HUASMCs, human umbilical artery smooth muscle cells. 
A

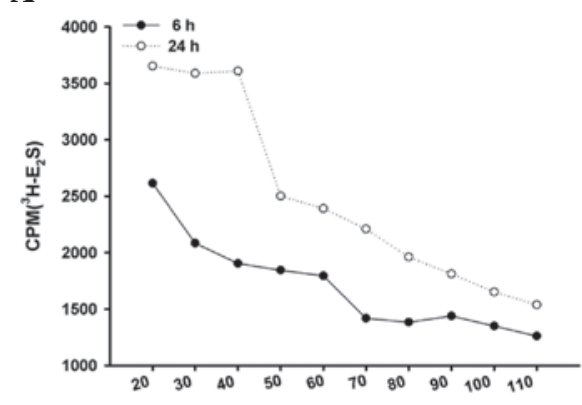

$17 \beta$-Estradiol (nM)
B

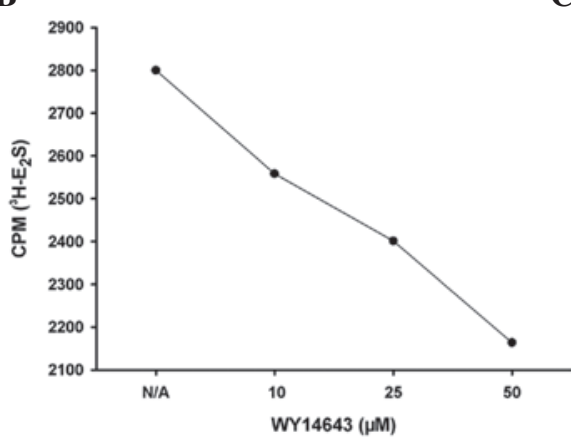

C

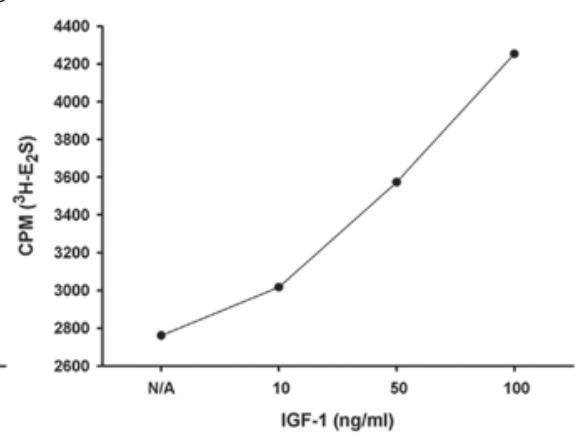

Figure 4. Effects of PPAR- $\alpha$ agonist WY14643 and IGF-1 on the activity of SULT1E1 in HUASMCs. (A) $10 \mathrm{nM}{ }^{3} \mathrm{H}-\mathrm{estradiol}$ combined with $10-100 \mathrm{nM}$ cold estradiol was added to untreated HUASMCs. Aliquots $(50 \mu \mathrm{l})$ of the medium were sampled at 6 or $24 \mathrm{~h}$. The counts of the aqueous phase ( $\left.\mathrm{E}_{2} \mathrm{~S}\right)$ following chloroform extraction represented SULT1E1 activity. HUASMCs treated with varying doses of (B) WY14643 or (C) IGF-1 for 24 h were incubated with $40 \mathrm{nM}$ estradiol $\left(30 \mathrm{nM}\right.$ estradiol and $10 \mathrm{nM}{ }^{3} \mathrm{H}$-estradiol) for a further $6 \mathrm{~h}$. The sulfated estradiol in the aliquots $(50 \mu 1)$ of the medium was calculated by liquid scintillation counting. PPAR- $\alpha$, peroxisome proliferator-activated receptor- $\alpha$; IGF-1, insulin-like growth factor-1; SULTIE1, estrogen sulfotransferase; HUVECs, human umbilical vein endothelial cells; HUASMCs, human umbilical artery smooth muscle cells.

A

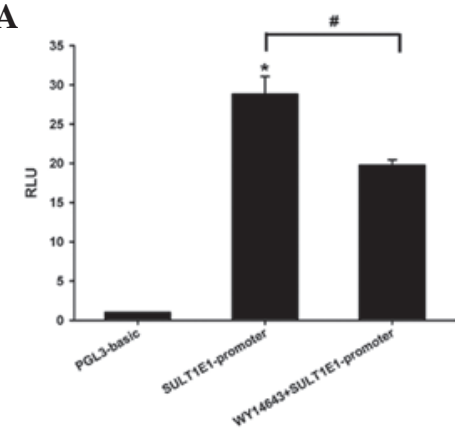

B

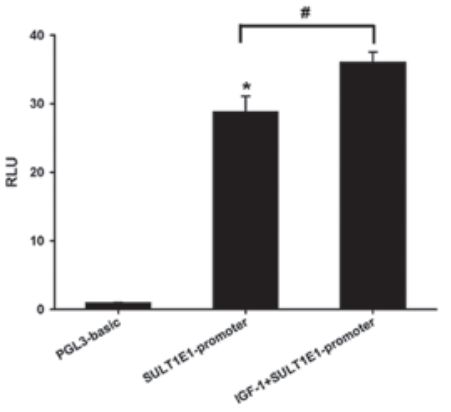

Figure 5. Effects of PPAR- $\alpha$ agonist WY14643 and IGF-1 on the activity of the SULT1E1 promoter in HEK-293 cells. HEK-293 cells were transfected with pGL3-basic or a SULT1E1-promoter for $48 \mathrm{~h}$ and cells were treated with (A) PPAR- $\alpha$ agonist WY14643 or (B) IGF-1 for $24 \mathrm{~h}$. ${ }^{\mathrm{P}}<0.05$ compared with the pGL3-basic vector group; ${ }^{\# P}<0.05$ compared with the pGL3-SULT1E1 promoter vector group. PPAR- $\alpha$, peroxisome proliferator-activated receptor- $\alpha$; IGF-1, insulin-like growth factor-1; SULTIE1, estrogen sulfotransferase; HEK-293, human embryonic kidney 293.

in the HUVECs and the HUASMCs (Fig. 2A and C); while IGF-1 significantly increased the SULTIE1 mRNA levels (Fig. 2B and D).

Effects of PPAR- $\alpha$ agonist WY14643 and IGF-1 on SULT1E1 protein levels in HUVECs and HUASMCs. In order to confirm the regulatory role of PPAR- $\alpha$ agonist WY14643 and IGF-1 in SULT1E1 expression, SULT1E1 protein levels were analyzed using western blotting. Varying concentrations of PPAR- $\alpha$ agonist WY14643 and IGF-1 were added to the HUVECs (Fig. 3A-D) or HUASMCs (Fig. 3E-H) for $24 \mathrm{~h}$.
The expression of SULT1E1 was downregulated by WY14643 (Fig. 3A and C), but upregulated by IGF-1, in the HUVECs (Fig. 3B and D). The expression pattern of SULT1E1 protein levels in HUASMCs was similar to that in HUVECs following treatment with the PPAR- $\alpha$ agonist WY14643 (Fig. 3E and G) and IGF-1 (Fig. 3F and H).

Effects of PPAR- $\alpha$ agonist WY14643 and IGF-1 on the activity of SULT1E1 in HUASMCs. SULT1E1 activity was measured following treatment with WY14643 or IGF-1 in HUASMCs. As shown in Fig. 4A, $40 \mathrm{nM}$ estradiol (30 $\mathrm{nM}$ estradiol and $10 \mathrm{nM}{ }^{3} \mathrm{H}$-estradiol) was capable of complete sulfation to $\mathrm{E}_{2} \mathrm{~S}$ in untreated HUASMCs following incubation with ${ }^{3} \mathrm{H}$-estradiol for $24 \mathrm{~h}$. However, $40 \mathrm{nM}$ estradiol was a sufficient quantity of substrate for estradiol sulfation within a $6 \mathrm{~h}$ incubation period. Therefore, $6 \mathrm{~h}$ was selected as the incubation period for the subsequent experiments. HUASMCs were treated with varying concentrations of WY14643 or IGF-1 for $24 \mathrm{~h}$ and were then incubated with $40 \mathrm{nM}$ estradiol $(30 \mathrm{nM}$ estradiol and $10 \mathrm{nM}{ }^{3} \mathrm{H}$-estradiol) for a further $6 \mathrm{~h}$. The sulfated estradiol decreased in cells treated with WY14643, but increased in the presence of IGF-1 (Fig. 4B and C), which suggests that WY14643 inhibited, whereas IGF-1 enhanced, the activity of SULT1E1 in the HUASMCs.

Effect of the PPAR- $\alpha$ agonist WY14643 and IGF-1 on the activity of the SULTIE1 promoter in HEK-293 cells. In order to clarify the regulatory mechanism of WY14643 and IGF-1 on SULT1E1 activity, the luciferase assay was performed using a Dual-Luciferase reporter assay system in HEK-293 cells. The activity of the SULT1E1 promoter increased 30-fold compared with the pGL3-basic vector. PPAR- $\alpha$ agonist WY14643 decreased, while IGF-1 increased, the luciferase activity of the SULT1E1 promoter (Fig. 5). The data suggested that WY14643 and IGF-1 regulated SULT1E1 expression at the transcriptional level.

\section{Discussion}

Estrogens exert potent atheroprotective effects by attenuating vascular dysfunctions. Estrogen metabolism is involved in the 
regulation of estrogen activity and affects vascular function. SULT1E1 is the predominant enzyme responsible for the sulfation of $\beta$-estradiol and estrone (21). According to our previous studies and the present study, SULT1E1 is abundantly expressed in HUVECs and HUASMCs. This suggests that vascular function is associated with the expression and activity of SULT1E1. This study demonstrated that the PPAR- $\alpha$ agonist WY14643 downregulated, but IGF-1 upregulated, the expression and activity of SULT1E1 in vascular cells. Since the SULTIE1 mRNA levels and luciferase activity of the SULT1E1 promoter were decreased by WY14643 and increased by IGF-1 treatment, we hypothesize that the PPAR- $\alpha$ agonist WY14643 and IGF-1 regulated SULT1E1 expression at the transcriptional level.

Increasing amounts of evidence indicate that PPAR- $\alpha$ agonists improve vascular function independent of their hypolipidemic effects (12). The underlying mechanisms include inhibiting inflammatory cell recruitment and activation, attenuating inflammatory responses (22), increasing cholesterol efflux and plaque stability, decreasing foam cell formation (23), vasoconstriction and thrombosis, and controlling vascular smooth muscle cell proliferation (24). In this study, we observed that the PPAR- $\alpha$ agonist WY14643 inhibited the expression and activity of SULT1E1 in vascular cells. Therefore, the PPAR- $\alpha$ agonist may increase the concentration and activity of estrogen by reducing the expression of SULT1E1. Less 17 $\beta$-estradiol was sulfated in HUASMCs treated with WY14643 compared with untreated cells.

Activation of PPAR- $\alpha$ is initiated by the binding of an agonist. Following ligand activation, PPAR- $\alpha$ forms heterodimers with retinoid $X$ receptor (RXR). The PPAR-RXR complex subsequently recognizes and binds to PPAR-response elements (PPREs) on target gene promoters, thereby activating their expression (25). Furthermore, the activation of the PPAR-RXR complex participates in the negative regulation of certain genes, either by processes independent of DNA-binding via interference with other signaling pathways, or by a DNA-dependent process involving the recruitment of co-repressors (26). Based on the data of the RT-qPCR assay and SULT1E1 promoter reporter activity assay, WY14643 regulated the expression and activity of SULTIE1 in vascular cells at the transcriptional level. However, the precise molecular mechanism of this repression requires further investigation.

IGF-1 exerts an anti-atherogenic effect. In endothelial cells, IGF-1 enhances the production of nitric oxide by endothelial nitric oxide synthase (eNOS) (27). In smooth muscle cells, IGF-1 prevents oxidative stress-induced apoptotic cell death and stimulates smooth muscle cell proliferation, migration and extracellular matrix synthesis, indicating a role for IGF-1 in the maintenance of plaque stability (28). According to our data, SULT1E1 expression and activity were enhanced by IGF-1. Once IGF-1 reduces the concentration of estrogen by increasing SULT1E1 activity, the anti-atherogenic role of estrogen may be attenuated. The actions of IGF-1 are mediated by the specific membrane receptor IGF-1R, a tyrosine kinase that undergoes autophosphorylation and catalyzes the phosphorylation of insulin receptor substrate proteins. Upon phosphorylation, insulin receptor substrate proteins interact with signaling molecules, including Akt, Ras/Raf and Rac (29).
The precise molecular mechanism that underlies the increase in SULT1E1 expression by IGF-1 at the transcriptional level requires further investigation.

In conclusion, this study investigated the effects of two important mediators of the process of atherosclerosis, a PPAR- $\alpha$ agonist and IGF-1, on the expression and activity of SULT1E1 in vascular cells. Thus, the PPAR- $\alpha$ agonist and IGF-1 may regulate the levels of active estrogens in endothelial cells and smooth muscle cells, thereby affecting the physiology and pathophysiology of the vascular walls.

\section{Acknowledgements}

This work was supported by The National Natural Science Foundation of China (NSFC 81030014, NSFC 81270497 and NSFC 81101762).

\section{References}

1. Nofer JR: Estrogens and atherosclerosis: insights from animal models and cell systems. J Mol Endocrinol 48: R13-R29, 2012.

2. Ishibashi H, Suzuki T, Suzuki S, et al: Estrogen inhibits cell proliferation through in situ production in human thymoma. Clin Cancer Res 11: 6495-6504, 2005.

3. Xu Y, Liu X, Guo F, et al: Effect of estrogen sulfation by SULT1E1 and PAPSS on the development of estrogen-dependent cancers. Cancer Sci 103: 1000-1009, 2012.

4. Tong MH, Jiang H, Liu P, Lawson JA, Brass LF and Song WC: Spontaneous fetal loss caused by placental thrombosis in estrogen sulfotransferase-deficient mice. Nat Med 11: 153-159, 2005.

5. Wada T, Ihunnah CA, Gao J, et al: Estrogen sulfotransferase inhibits adipocyte differentiation. Mol Endocrinol 25: 1612-1623, 2011.

6. Gong H, Jarzynka MJ, Cole TJ, et al: Glucocorticoids antagonize estrogens by glucocorticoid receptor-mediated activation of estrogen sulfotransferase. Cancer Res 68: 7386-7393, 2008.

7. Kodama S, Hosseinpour F, Goldstein JA and Negishi M: Liganded pregnane $\mathrm{X}$ receptor represses the human sulfotransferase SULT1E1 promoter through disrupting its chromatin structure. Nucleic Acids Res 39: 8392-8403, 2011.

8. Fruchart JC: Peroxisome proliferator-activated receptor-alpha (PPARalpha): at the crossroads of obesity, diabetes and cardiovascular disease. Atherosclerosis 205: 1-8, 2009.

9. Hennuyer N, Tailleux A, Torpier G, et al: PPARalpha, but not PPARgamma, activators decrease macrophage-laden atherosclerotic lesions in a nondiabetic mouse model of mixed dyslipidemia. Arterioscler Thromb Vasc Biol 25: 1897-1902, 2005.

10. Srivastava RA: Evaluation of anti-atherosclerotic activities of PPAR-alpha, PPAR-gamma, and LXR agonists in hyperlipidemic atherosclerosis-susceptible F(1)B hamsters. Atherosclerosis 214: 86-93, 2011.

11. Zahradka P, Yurkova N, Litchie B, Moon MC, Del Rizzo DF and Taylor CG: Activation of peroxisome proliferator-activated receptors alpha and gammal inhibits human smooth muscle cell proliferation. Mol Cell Biochem 246: 105-110, 2003.

12. Hamblin M, Chang L, Fan Y, Zhang J and Chen YE: PPARs and the cardiovascular system. Antioxid Redox Signal 11: 1415-1452, 2009.

13. Fang HL, Strom SC, Cai H, Falany CN, Kocarek TA and Runge-Morris M: Regulation of human hepatic hydroxysteroid sulfotransferase gene expression by the peroxisome proliferator-activated receptor alpha transcription factor. Mol Pharmacol 67: 1257-1267, 2005.

14. Higashi Y, Sukhanov S, Anwar A, Shai SY and Delafontaine P: IGF-1, oxidative stress and atheroprotection. Trends Endocrinol Metab 21: 245-254, 2010.

15. Higashi Y, Sukhanov S, Anwar A, Shai SY and Delafontaine P: Aging, atherosclerosis, and IGF-1. J Gerontol A Biol Sci Med Sci 67: 626-639, 2012.

16. Han Y, Qi Y, Kang J, Li N, Tian X and Yan C: Nerve growth factor promotes formation of lumen-like structures in vitro through inducing apoptosis in human umbilical vein endothelial cells. Biochem Biophys Res Commun 366: 685-691, 2008. 
17. Vadiveloo PK, Filonzi EL, Stanton HR and Hamilton JA: G1 phase arrest of human smooth muscle cells by heparin, IL-4 and cAMP is linked to repression of cyclin D1 and cdk2. Atherosclerosis 133: 61-69, 1997.

18. Yeong P, Ning Y, Xu Y, Li X and Yin L: Tryptase promotes human monocyte-derived macrophage foam cell formation by suppressing LXRalpha activation. Biochim Biophys Acta 1801: 567-576, 2010

19. Wang X, Spandidos A, Wang H and Seed B: PrimerBank: a PCR primer database for quantitative gene expression analysis, 2012 update. Nucleic Acids Res 40: D1144-D1149, 2012.

20. Kushida A, Hattori K, Yamaguchi N, Kobayashi T, Date A and Tamura H: Sulfation of estradiol in human epidermal keratinocyte. Biol Pharm Bull 34: 1147-1151, 2011.

21. Xu Y, Yang X, Wang Z, et al: Estrogen sulfotransferase (SULT1E1) regulates inflammatory response and lipid metabolism of human endothelial cells via PPARgamma. Mol Cell Endocrinol 369: 140-149, 2013.

22. Lalloyer F, Wouters K, Baron M, et al: Peroxisome proliferatoractivated receptor-alpha gene level differently affects lipid metabolism and inflammation in apolipoprotein E2 knock-in mice. Arterioscler Thromb Vasc Biol 31: 1573-1579, 2011.

23. Chinetti G, Lestavel S, Bocher V, et al: PPAR-alpha and PPAR-gamma activators induce cholesterol removal from human macrophage foam cells through stimulation of the ABCA1 pathway. Nat Med 7: 53-58, 2001.
24. Gizard F, Nomiyama T, Zhao Y, et al: The PPARalpha/p16INK4a pathway inhibits vascular smooth muscle cell proliferation by repressing cell cycle-dependent telomerase activation. Circ Res 103: 1155-1163, 2008.

25. Plutzky J: The PPAR-RXR transcriptional complex in the vasculature: energy in the balance. Circ Res 108: 1002-1016, 2011.

26. Zoete V, Grosdidier A and Michielin O: Peroxisome proliferator-activated receptor structures: ligand specificity, molecular switch and interactions with regulators. Biochim Biophys Acta 1771: 915-925, 2007.

27. Sukhanov S, Higashi Y, Shai SY, et al: Differential requirement for nitric oxide in IGF-1-induced anti-apoptotic, anti-oxidant and anti-atherosclerotic effects. FEBS Lett 585: 3065-3072, 2011.

28. Wang J, Razuvaev A, Folkersen L, et al: The expression of IGFs and IGF binding proteins in human carotid atherosclerosis, and the possible role of IGF binding protein-1 in the regulation of smooth muscle cell proliferation. Atherosclerosis 220: 102-109, 2012.

29. Delafontaine P, Song YH and Li Y: Expression, regulation, and function of IGF-1, IGF-1R, and IGF-1 binding proteins in blood vessels. Arterioscler Thromb Vasc Biol 24: 435-444, 2004. 